\title{
Studies on effects of different solvent extracted samples of $O$. sanctum (Basil) stem on the growth of S. typhi
}

*Seema Kumari and Vinod Prasad

Department of Botany,

B.N. College, Patna University,

PATNA, INDIA.

*Corresponding Author

E-mail : Seemakumaribt@gmail.com

Received : 21.09.2020; Accepted : 15.11.2020

\begin{abstract}
Typhoid is a problematic fever, caused by Salmonella typhi. Present investigation is based upon studies of antibacterial effects of different solvent extracted samples of $O$. sanctum stem on growth of $S$. typhi. The crude extracts of stem of $O$. sanctum were prepared from three different solvents such as methanol, ethyl acetate and hot water. Four different concentrations $(25 \%$, $50 \%, 75 \%$ and $100 \%$ ) were prepared from each one of the solvent extracted sample. Agar well diffusion method was used to test the antibacterial activity of different solvent extracted samples of $O$. sanctum stem. Analysis of data indicates that a metabolic extract of $O$. sanctum stem shows maximum zone of inhibition and it was of $26 \mathrm{~mm}$ at $100 \%$ concentration and minimum zone of inhibition was of $19 \mathrm{~mm}$ at $50 \%$ concentration. Ethyl acetate extract of $O$. sanctum stem shows maximum zone of inhibition and it was of $29 \mathrm{~mm}$ at $100 \%$ concentration and minimum was of $14 \mathrm{~mm}$ at $25 \%$ concentration. Hot water extract of $O$. sanctum stem shows maximum zone of inhibition and it was of $28 \mathrm{~mm}$ at $100 \%$ concentration and minimum zone of inhibition was of $12 \mathrm{~mm}$ at $25 \%$ concentration. This study indicates that ethyl acetate extract of 0 . sanctum stem is more effective against $S$. typhi as compared to methanol and hot water extracted solvent. Contrary to the antibiotics or synthetic medicine associated with many side effects, this plant part can be used as a herbal medicines against Salmonella typhi infection.
\end{abstract}

Figures : $02 \quad$ References : 10

Table : 00

KEY WORDS : O. sanctum, S. typhi, Solvent, Zone of inhibition.

\section{Introduction}

Plants are the richest source for the development of conventional as well as modern medicines since thousands of years and a large number of drugs are synthesized from medicinal plants ${ }^{3}$. Medicinal plants are plants which constitute bioactive substances or phytochemicals which are the precursors for the synthesis of useful drugs and treatment of different ailments. The use of plant and plant products or parts as medicine is as old as the beginning of human civilization. Among the 7000 , species of medicinal plants recognized all over the world only few of them are used for their medicinal value ${ }^{4}$. The reported data available on medicinal plants are comparatively less and the study of vast majority of plants is still unknown. Therefore there is need to still in search of medicinal plants and plant products. Today drug safety remains a global issue to treat infectious disease. This herbal preparation is lower incidence of adverse drug reaction as compared to modern medicine. The reduced cost of herbal medicine encourage both public and health care institutions to consider plant medicines as alternative to synthetic drug ${ }^{5}$.Thus the aim of our experiment is to study the effect of different solvent extracted samples of O. sanctum stem on the growth of S. typhi. S.typhi, a rod shaped flagellated gram negative bacteria that causes systemic infection and typhoid fever in human. . Typhoid fever is a worldwide public health problem in developing countries to lack of hygiene and polluted drinking water ${ }^{6,7}$. According to World health organization reports, approximately 21 million cases are reported per year and out of them 222000 people die from this disease. ${ }^{10}$ It causes many death in developing countries and spread through contaminated water and food. The plant that is used in our experiment is O. sanctum stem. O. sactum belongs to the family Lamiaceae and its vernacular name is Tulsi in Hindi and holy Basil in English. The medicinal use of tulsi is known from thousands of years and also this plant play an important role in maintaining human health ${ }^{8}$. It is found that the different extracts of tulsi and essential oil possess antibacterial activity ${ }^{9}$. The different extracts of O.sanctum show antimicrobial properties against gram positive and gram negative bacteria.O. sanctum is used for the treatment of infectious diseases caused by bacteria, virus, fungi and insect without showing any side effect ${ }^{2}$. That's why these properties made this plant unique from other medicinal plants. Thus the present study involved to explore the antibacterial activity of medicinal plant O. sanctum stem 

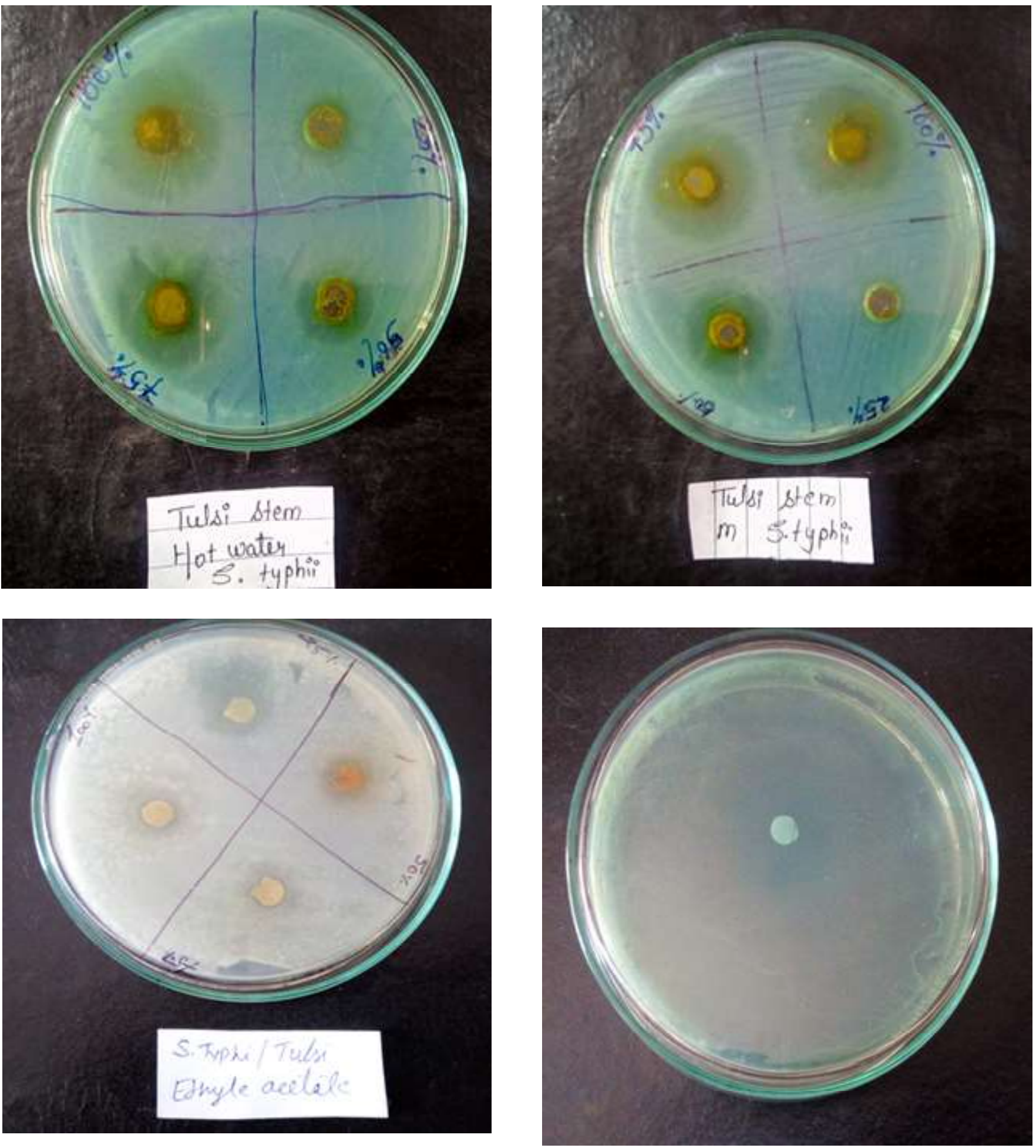

Fig. 1 : Diameter of ZOI of standard antibiotic ciprofloxacin against S.typhi

against S.typhi.

\section{Materials and Method}

Collection of plant material: The medicinal plant used for the experiment was Fresh stem of O.sanctum. The plant part was collected from the B.N. College campus, Ashok Raj path, Patna University, Patna. The plant parts were identified according to various literatures.
Microorganism used: The bacterial culture used in present study included S. typhi and were received from ATCC. (ATCC No 6539)

Culture maintenance: The bacterial strain was maintained in nutrient agar media at $37^{\circ} \mathrm{C}$ for 24 hours. The stock culture slants were maintained at $4^{\circ} \mathrm{C}$.

Plant extract preparation:, Three different 


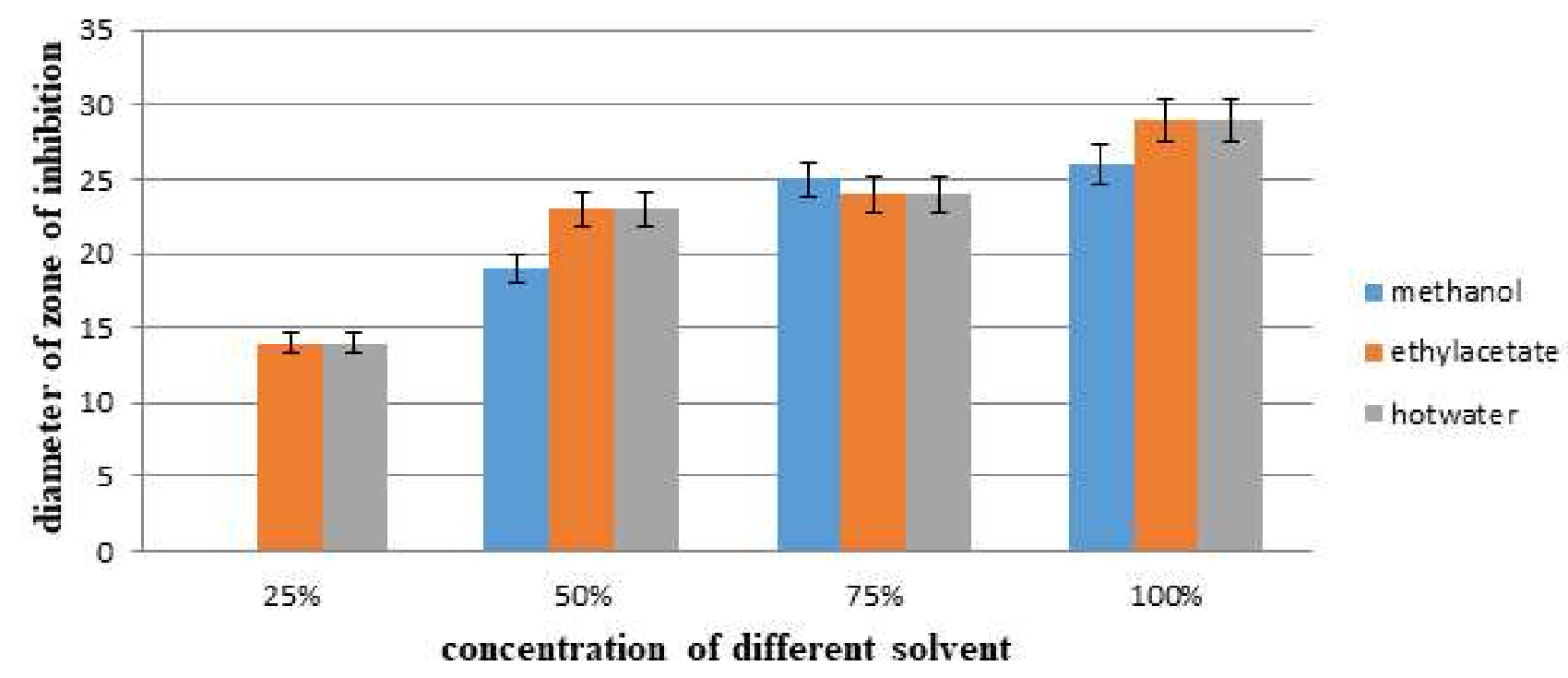

Fig. 2 : Effect of different solvent extracted sample on a growth of S. typhi

solvents viz., methanol, ethyl acetate and hot water were used for the preparation of crude extract of. O. sanctum stem.

Different size of previously cut stem part of O. sanctum was loaded in the thimble of Soxhlet apparatus. It was fitted with appropriate size round bottle flask with $250 \mathrm{ml}$ of each solvent and upper part was fitted with condenser. Constant heat was provided by Mantox heater for recycling of the solvent. After complete extraction, the extract in round bottle flask was transferred into clean and preweighed universal tubes. Universal tubes containing extract were weighted and noted down and finally, the percentage yield was calculated. Percentage yield was as dividing initial weight of raw material taken by final weight of extract.

Kirby Bauer disc diffusion technique was used to test the antimicrobials effect of different solvent extracted sample O.sanctum stem. Disc were impregnated with different solvent extracts at different concentration ranging from $25-100 \%$. Nutrient agar was prepared and autoclaved at $15 \mathrm{lbs}$ pressure for 20 minutes and cooled at $45^{\circ} \mathrm{C}$. The cooled media was poured on to sterile petriplate and allowed for solidification. The plate with media was seeded with S.typhi suspension using sterile sweber. Discs were placed on the four corner of each petriplate. Standard antibiotics ciprofloxacin were placed on the other petridish. Petri dishes were incubated at $37^{\circ} \mathrm{C}$ for 24 hours. After incubation diameter of zone of inhibition around the petridish were measured and expressed in $\mathrm{mm}$.

\section{Result and Discussion}

Analysis of data indicates that a methanolic extract of O.sanctum stem shows maximum zone of inhibition. It was of $26 \mathrm{~mm}$ at $100 \%$ concentration and minimum zone inhibition was of $00 \mathrm{~mm}$ at $25 \%$ concentration. Ethyl acetate extract of $O$. sanctum stem shows maximum zone of inhibition. It was of $29 \mathrm{~mm}$ at $100 \%$ concentration and minimum zone of inhibition was of $14 \mathrm{~mm}$ at $25 \%$ concentration. Hot water extract of O.sanctum stem shows maximum zone of inhibition. It was of $29 \mathrm{~mm}$ at $100 \%$ concentration. Minimum zone of inhibition was of $14 \mathrm{~mm}$ at $25 \%$ concentration. The standard antibiotics ciprofloxacin was used against S.typhi growth. This antibiotics shows zone of inhibition diameter $29 \mathrm{~mm}$.

This study indicates that ethyl acetate and hot water extracted sample of O.sanctum stem is more effective against S.typhi infection as compared to methanolic extracted solvent of O.sanctum stem . Contrary to the antibiotics or antibacterial medicine associated with many side effects, this plant part can be used as a herbal medicine against $S$. typhi infections. In conclusion, the effect of different solvent extracted samples of $O$. sanctum stem on the growth of S.typhi warrants the exploration of an alternative natural antimicrobials agent. Medicinal plants have become the focus of intense study in terms of validation of their traditional uses and the determination of their authentic pharmacological effects. Therefore there is need to search for new infections fighting strategies to control these microbial infection.

The present study suggests using this plant part to stop the growth of S.typhi infection. Thus there is a scope for using ethyl acetate and hot water extract of O.sanctum stem against S.typhi infection. 


\section{References}

1. Akerele O. Summary of WHO Guidelines for the Assessment of Herbal Medicines Herbal Gram. 1993; 22: 13-28.

2. Devi UP, Gonasoundari A, Vrinda B, Srinivasan KK, Unnikrishanan MK. Radiation protection by the Ocimum sanctum flavonoids orientin and vicenin: Mechanism of action, Radiat Res. 2000; 154(4): 455-460.

3. Hammer A, Carson CF, Riley TV. Antimicrobial activity of essential oils and other plant extracts. J. Appl. Microbiol. 1999; 86(6):985.

4. Manandhar NP. Plants and People of Nepal. Timber Press, USA. 2000; p.50.

5. Nair R, Kalariya T, Chanda S. Antibacterial activity of some selected Indian medicinal flora. Turk. J. Biol. 2005; $29: 41-47$.

6. Ni Y, Springer MJ, Guo J, Finger-Baker I, Wilson JP, Cobb RR, et al. Development of a synthetic Vi polysaccharide vaccine for typhoid fever. Vaccine 2017; 35 (51): -7121-7126.

7. Ochiai RL, Acosta CJ, Danovaro-Holliday MC, Baiqing Bhattacharya SK, Agtini MD , et al. Domi Typhoid Study Group. A study of typhoid fever in five Asian countries: disease burden and implications for controls. Bull World Health Organ. 2008; 86 (4) : 260-268.

8. Rahman S, Islam R, Kamruzzaman M, Alam K, Jamel AHM. Ocimum sanctum L: A review of Phytochemical and pharmacological profile, Am J Drung Disc Develop. 2011, 6: 1-15.

9. Seth J, Sood S, Seth S, Talwar A. Protective effect of Tulsi (Ocimum sanctum) on lipid peroxidation in stress induced by anemic hypoxia in rabbits, Indian J. Physiol. Pharmacol. 2003 ; 47: 115-119.

10. World Health Organization. Immunization, vaccines and biologicals. Typhoid. 2017 [cited 2017 November 24] Available from: http://www.who.int/immunization/diseases/typhoid/en/. 